This document is the accepted manuscript version of the following article:

Carminati, M. C., Piazzese, C., Pepi, M., Tamborini, G., Gripari, P., Pontone, G., ... Caiani, E. G. (2018). A statistical shape model of the left ventricle from real-time 3D echocardiography and its application to myocardial segmentation of cardiac magnetic resonance images. Computers in Biology and Medicine, 96, 241-251.

https://doi .org/10.1016/j.compbiomed.2018.03.013

\title{
A statistical shape model of the left ventricle from real-time 3D echocardiography and its application to myocardial segmentation of Cardiac Magnetic Resonance images
}

Carminati MC*a ${ }^{*}$ Piazzese $C^{* b}$, Pepi $\mathrm{M}^{\mathrm{c}}$, Tamborini $\mathrm{G}^{\mathrm{c}}$, Gripari $\mathrm{P}^{\mathrm{c}}$, Pontone $\mathrm{G}^{\mathrm{c}}$, Krause $\mathrm{R}^{\mathrm{d}}$, Auricchio A , RM Lang $^{\mathrm{e}}$, Caiani $\mathrm{EG}^{\mathrm{f}}$

*the authors equally contributed to this work

${ }^{a}$ Laboratory for Neutron Scattering and Imaging, Paul Scherrer Institut, CH5232 Villigen-PSI, Switzerland

${ }^{\mathrm{b}}$ School of Engineering, Cardiff University, Cardiff, United Kingdom

${ }^{\mathrm{c}}$ Centro Cardiologico Monzino, IRCCS Milano, Italy

${ }^{\mathrm{d}}$ Centre for Computational Medicine in Cardiology, Institute of Computational Sciences, Università della Svizzera Italiana, Lugano, Switzerland

e Noninvasive Cardiac Imaging Laboratories, Department of Cardiology, University of Chicago, Chicago, Illinois, USA

${ }_{\mathrm{f}}^{\mathrm{f}}$ Department of Electronics, Information and Bioengineering, Politecnico di Milano, Milano, Italy

Corresponding author:

M.Chiara Carminati, PhD

Laboratory for Neutron Scattering and Imaging

Paul Scherrer Institut

WBBA/110

CH-5232 Villigen PSI, Switzerland

phone: +4156310 5162

email: chiara.carminati@psi.ch 


\section{Abstract}

Object: We present in this paper the application of a statistical shape model of the left ventricle (LV) built from transthoracic real time 3D echocardiography (3DE) to segment the LV endocardium and epicardium in cardiac magnetic resonance (CMR) images.

Material and Methods: The LV model was built from a training database constituted by over 9000 surfaces obtained from retrospectively selected 3DE examination of 435 patients with various pathologies. Three-dimensional segmentation of the endocardium and the epicardium was obtained by processing CMR images acquired in 30 patients with a dedicated active shape modelling (ASM) algorithm using the proposed LV model.

Results: The segmentation results obtained with the proposed method were compared with those obtained by the manual reference technique; similarity was proven by computing: i) point to surface distance $(<2 \mathrm{~mm})$, ii) Dice similarity coefficient (>89\%), iii) Hausdorff distance $(\sim 5 \mathrm{~mm})$. This was furthermore confirmed by equivalence testing, linear regression and Bland Altman analysis applied on derived clinical parameters, such as LV volumes and mass.

Conclusions: This study showed the potential usefulness of the proposed inter-modal ASM approach featuring a 3DEbased LV model for the 3D segmentation of the LV myocardium in CMR images.

\section{Keywords}

Magnetic Resonance Imaging, Cine; Echocardiography, Three-Dimensional; Multimodal Imaging; Image Processing, Computer-Assisted; Diagnostic Imaging 


\section{Introduction}

In recent years, model-based techniques have become a popular solution for 3D image segmentation and analysis [1-3] [4]. In this two-steps technique, first a model has to be built from a training set constituted of contours obtained by an expert observer, suitable to encode the shape and the statistical variability of an organ of interest. Then, the model is adapted to match the same organ in new images. Examples/instances of such techniques are represented by activeshape modeling (ASM, [5]) and active appearance models (AAM, [6]), where the shape is expressed as a point distribution model (PDM), i.e. a set of corresponding landmarks extracted from segmentation, and its allowed variations are usually determined by principal component analysis (PCA).

In cardiac imaging, model-driven applications have been proposed for the analysis of the left ventricle (LV) [7-13], both right and left ventricles [14-20], the four chambers [21] or the entire heart [22-25]. The segmentation of the cardiac chambers is a necessary requirement for the quantitative analysis of cardiac function and, despite the efforts in the development of semi-automated techniques $[3,26]$, in clinical practice this procedure is still performed manually. The adoption of methods that incorporate prior shape knowledge benefits from the fact that the shape of the cardiac ventricles with normal or altered function is approximately known and investigated in clinical studies, as the heart remodels by changing its shape in response of pathophysiological processes attempting to restore its normal function. Most model-based techniques were applied to the segmentation of cardiac magnetic resonance (CMR) images MRI [7, $9,10,11,14,16,18-21]$, as it is currently the reference modality for the non-invasive assessment of the LV volumes and systolic function. One main limitation of such approaches relies on the construction of models that include a suitable large training database where the cardiac chambers have to be accurately delineated. In most published research $[9,10,14,17,19,21,23,24]$, statistical models of shapes were created by processing less than 100 training set images with boundaries obtained by manual tracings.

The first contribution of this paper is the construction of a statistical shape model (SSM) of the LV endocardium built from a large database of surfaces obtained from semi-automatic segmentation of transthoracic real-time 3D echocardiography (3DE) and its application for 3D segmentation of the LV endocardium in CMR images. This concerns an expansion of our previous work [27], in which the proof of concept was presented.

Secondly, we propose a novel approach for the LV epicardial segmentation. We hypothesize that the same endocardial LV shape model may be reused to segment the LV epicardium in cardiac MRI. This was based on the assumption that the endocardium and the epicardium in the LV share similar shape characteristics and fostered by the limitation of constructing a specific LV epicardial model from 3DE datasets. 
We finally provide a quantitative evaluation of the accuracy of LV endocardial and epicardial segmentation performance by comparing it with the manual tracings reference technique on 30 CMR datasets in end-diastolic (ED) and end-systolic (ES) frames obtained from consecutive patients, including normal and failing hearts.

\section{Materials and Methods}

In this section, the methodological approach for model creation and image segmentation is described. As schematically represented in Figure 1, the approach can be summarized in the following step:

- Model construction of the LV from real-time 3D echo images;

- Segmentation of the endocardium in CMR images by adapting the proposed model, the manual definition of some anatomical landmarks is needed to compute the initial position and scale of the model;

- Segmentation of the epicardium in CMR images, using the same statistical model and the endocardial segmentation as initial pose.

\section{Model construction from 3DE segmented images}

The imaging database used in this study for the LV model construction was retrospectively collected from a clinical cohort of 435 subjects with various pathology (293 with normal LV function, 78 with dilated cardiomyopathy, 13 with aortic insufficiency, 11 with aortic stenosis, 28 with mitral regurgitation and 12 with mitral stenosis) that underwent 3DE examination at the University of Chicago, IL, USA or at Centro Cardiologico Monzino, Milano, Italy.

Demographic data are summarized in Table 1.

In both institutions, ECG-gated 3DE image acquisition was performed during breath-holding using a iE33 (Philips) ultrasound system with X3-1 probe, in wide-angled modality resulting in four to seven wedge-shaped sub-volumes $\left(93^{\circ} \times 21^{\circ}\right)$ over consecutive cardiac cycles.

3DE datasets were analyzed for LV volume quantification by using commercial software (4D LV analysis, TomTec Image Arena, München, Germany), featuring semi-automated endocardial segmentation. At the initialization step, the user is asked to manually select three anatomical landmarks in one frame considered as reference and set at ED by default: i) the center of the mitral valve (MV), ii) the apex position (AP) and iii) the center of the aortic valve (AO). The MV and the AP are selected on the three standard long-axis cut-planes, i.e. the apical 2-, 3- and 4 chambers, while the AV point is selected on a short-axis cut-plane at the level of the aortic valve. At this stage, the user is able to navigate through the volume and to change the reference frame considered for the point selection or the short axis cutplane considered for the AV point selection. Then, the software automatically segments the 3D LV endocardium and 
tracks it in all frames of the cardiac cycle, giving also the possibility of manually adjusting the detected contours. The tracked surfaces can be exported for further analysis in the form of triangulated meshes, each defined by 642 nodes and by its connectivity matrix.

The anatomical correspondence of nodes belonging to each surface was guaranteed from the segmentation process, and the nodes corresponding to the three anatomical landmarks (AP, MV and AO) selected by the user were uniquely identified on each surface and tracked through the cardiac cycle. In Figure 2, an example of the exported mesh is shown, together with the position of the AP, MV and AO nodes.

The a priori knowledge of the MV, AO and AP position was used for surface alignment. First, for each surface the center of mass was computed and translated to the point of coordinates $[0,0,0]$. The LV long axis $\left(v_{1}\right)$ was defined as the vector joining MV and AP, and similarly for the aortic axis $\left(v_{2}\right)$ considering MV and AO. For each surface, the local reference system was then defined as:

$$
\left\{\begin{array}{c}
\boldsymbol{v} \mathbf{1}=\frac{M V-A P}{|M V-A P|} \\
\boldsymbol{v} \mathbf{2}=\boldsymbol{v} \mathbf{1} \times \frac{A O-M V}{|A O-M V|} \\
\boldsymbol{v} \mathbf{3}=\boldsymbol{v} \mathbf{1} \times \boldsymbol{v} \mathbf{2}
\end{array}\right.
$$

The long axis (v1) of all the meshes was rotated to customarily match the $\mathrm{z}$-axis of the reference system $([0,0,1])$, and rotation around v1 was achieved by matching v2 to one randomly selected surface. Scaling was not performed on purpose, to include this anatomical variability in the statistical model [28]. After shape alignment, each $\mathrm{i}_{\text {th }}$ surface was described by a concatenated vector $\mathrm{x}$ comprising all $\mathrm{k}$ coordinates:

$$
\boldsymbol{x}_{i}=\left[x_{1 i}, y_{1 i}, z_{i 1}, x_{2 i}, y_{2 i}, z_{2 i}, \ldots, x_{k i}, y_{k i}, z_{k i}\right]^{\prime}
$$

The mean model was then computed by averaging the coordinates of the corresponding points of all $\mathrm{N}$ surfaces constituting the training set:

$$
\widehat{\boldsymbol{x}}=\frac{1}{N} \sum_{i=1}^{N} x_{i}
$$

and its covariance matrix was obtained:

$$
\boldsymbol{S}=\frac{1}{N-1} \sum_{i=1}^{N}\left(\boldsymbol{x}_{\boldsymbol{i}}-\widehat{\boldsymbol{x}}\right)\left(\boldsymbol{x}_{i}-\widehat{\boldsymbol{x}}\right)^{T}
$$


From S, the eigenvectors and eigenvalues of the training set are computed using principal component analysis (PCA). The first $\phi_{\mathrm{i}}$ eigenvectors associated to the $\mathrm{t}=92$ largest eigenvalues, describing $99.7 \%$ of the variability, were retained to reduce the system dimension, and they were represented by the matrix $\phi=\left(\phi_{1}, \phi_{2}, \ldots \phi_{t}\right)$. Figure 3 shows the resulting first two modes of variations of the obtained SSM as mean plus and minus 1.5 and 3 standard deviations.

\section{Application to LV segmentation in CMR datasets}

\subsection{CMR pre-processing}

The application of the SSM for the 3D LV segmentation of CMR datasets was performed considering simultaneously the CMR long-axis (LA) images and the stack of short-axis (SA) images. First, CMR images were pre-processed to compensate for misalignments due to the non-perfectly repeatable level of apnea during acquisition, by applying the method described in [29]. Briefly, first the alignment of the 2 chambers $(2 \mathrm{CH})$ and 4 chambers $(4 \mathrm{CH}) \mathrm{LA}$ view is visually checked and manually adjusted, if necessary. Then the 3D position of the SA images is automatically corrected by maximizing the normalized cross correlation of pixel intensities at SA-LA plane intersections. This step is necessary to avoid distortion in the segmented 3D LV surface.

\subsection{Initial Pose and Scale}

An initialization step was achieved by the manual selection of points corresponding to anatomical landmarks on LA CMR images.

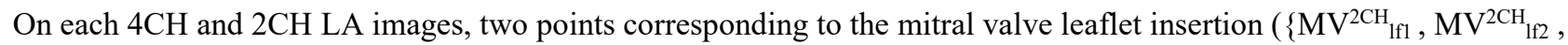
$\mathrm{MV}^{4 \mathrm{CH}}{ }_{1 \mathrm{f}}, \mathrm{MV}^{4 \mathrm{CH}_{1 \mathrm{f}}}$ \}, where lf1 and lf2 refer to the left and right leaflet insertion, respectively) and one point at the $\mathrm{LV}$ apex were selected $\left(\left\{\mathrm{AP}^{2 \mathrm{CH}}, \mathrm{AP}^{4 \mathrm{CH}}\right\}\right)$.

From these points, approximate positions of MV center and the apex in CMR reference system were obtained by averaging the respective point's coordinates:

$$
\begin{aligned}
& M V_{C M R}=\operatorname{mean}\left(M V_{l f 1}^{2 C H}, M V_{l f 2}^{2 C H}, M V_{l f 1}^{4 C H}, M V_{l f 2}^{4 C H}\right) \\
& A P_{C M R}=\operatorname{mean}\left(A P^{2 C H}, A P^{4 C H}\right)
\end{aligned}
$$

The $\mathrm{LV}$ axis was computed as the straight line connecting $\mathrm{MV}_{\mathrm{CMR}}$ and $\mathrm{AP}_{\mathrm{CMR}}$, and the $\mathrm{LV}$ center of mass obtained as the average position between these two points. On the 3-chambers $(3 \mathrm{CH}) \mathrm{LA}$ image, one point was selected in correspondence to the center of the aortic valve $\left(\mathrm{AO}_{\mathrm{CMR}}\right)$. From $\mathrm{MV}_{\mathrm{CMR}}, \mathrm{AP}_{\mathrm{CMR}}$ and $\mathrm{AO}_{\mathrm{CMR}}$, the $\mathrm{CMR}$ reference system was computed, as expressed similarly in Eq. 1.

The aim of this manual initialization was threefold. First, it allowed an initial alignment of the mean shape to the CMR 
data to be analyzed by matching the center of mass coordinates and the LV orientation, expressed by Eq1. Second, a scale factor was computed as the ratio between the mitral valve and apex distance computed in CMR data $\left(\mathrm{MV}_{\mathrm{CMR}}-\right.$ $\left.\mathrm{AP}_{\mathrm{CMR}}\right)$ and in the mean shape $\left(\mathrm{MV}_{\mathrm{SSM}}-\mathrm{AP}_{\mathrm{SSM}}\right)$, and applied to the mean model, if inside the allowable variations defined in the ASM as the $\pm 3 \sigma$ (standard deviation) range around the mean SSM. Finally, it allowed the automatic selection of the SA planes in the image stack to be used for LV endocardial segmentation, while excluding sub-apical and atrial slices whose 3D coordinates were below or above the computed mitral valve plane or the apex position, respectively. At this stage, the number of CMR SA slices that will be used for the LV 3D segmentation can be corrected by the user through an interactive GUI, by including or excluding planes where appropriate.

\subsection{Endocardial segmentation}

Segmentation was performed according to the ASM approach by iteratively searching new instances $\mathbf{x}_{\mathbf{n}}$ of the shape $\mathbf{x}$ by varying the shape parameters b according to: $\overrightarrow{\boldsymbol{x}}_{n}=T(\overrightarrow{\boldsymbol{x}}+\Phi \boldsymbol{b})$, where $\mathrm{T}$ is a similarity transform, $\phi$ is the matrix containing the eigenvectors corresponding to the main $\mathrm{t}$ axes of variation, and $\mathrm{b}$ is a $\mathrm{t}$-dimensional weighting vector containing the model parameters, varying within limits prescribed by the allowable shape domain $( \pm 3 \sigma)$ to ensure new shapes to be valid. After the initial model pose, the application of ASM as a deformable template for image segmentation involves the following steps: i) feature point search, ii) pose parameters calculation and iii) shape parameters estimation [1].

Feature point search was performed for CMR images as follows. The intersections between the model and the CMR planes were computed: for each mesh triangle crossed by the SA and LA planes, intersection coordinates were obtained by linear interpolating the triangle perimetral segments, thus obtaining two points for each triangle. Furthermore, an additional point placed halfway between the so-obtained couple of points was considered, in order to expand the research domain (Figure $4 \mathrm{a})$ and b) ).

Pixel intensity profiles of the line segments directed along the LV radial direction, i.e. from the center of mass of all intersections to each computed intersection (Figure $4 \mathrm{c}$ ), were computed and systematically aligned to generate a 2D image, named $L$, constituting the research space (Figure 4 d). For SA images, the length of each radial profile was dependent on the slice position along the LV and it spanned from the halfway position between each intersection points and their centroid to the point placed at a radial distance corresponding to $7 \mathrm{~mm}$ outside the model. This outer width was automatically doubled (14 mm), if a new feature point was not found. Following this scheme, the dimensions of $L$ for SA images were decreasing from the base to the apex, with size ranging about 40-150 and 20-60 mm along the two 
directions. For $2 \mathrm{CH}$ and $4 \mathrm{CH}$ LA images, the radial sampling followed a similar strategy but with a fixed line length of $30 \mathrm{~mm}$, centered at image/model intersections.

The research space $L$ was subsequently processed by a k-means clustering approach, featuring five clusters to represent blood pool, myocardium and external structures (Figure 4 e): two clusters were chosen to represent intensities belonging to the blood pool, and the remaining three for pixels of darker intensity in the myocardium From L, a binary image was obtained by grouping together the brighter clusters (cluster 1 and 2) and the darker ones (cluster 3,4,5) to define the endocardial interface (Figure 4, f)), namely the transition between the LV cavity and all others structures. Then, morphological and derivative-based smoothing operations were applied to prevent the presence of erroneous points due to papillary muscles, artifacts or weak features (Figure $4, \mathrm{~g}$ )). The interface defined as the pixel coordinates in $\mathrm{L}$ at white-black transition was then extracted and re-transformed into the CMR reference system. Finally, in order to include the papillary muscles in the LV cavity, each set of pixel positions detected at blood-pool/myocardial transition was processed to find the smallest convex set that contains the points. The border pixels of the convex hull were selected as feature points. For the LA images, the same k-means clustering and processing were applied for the feature points search, with the exception of the mitral valve region, where the feature points were defined as those constituting the line segment connecting the points at leaflets insertions selected during the initialization step.

Pose parameters were estimated first by deriving the rigid transformation matrix $\mathrm{T}$ necessary to align the model $\mathbf{x}$ to the detected feature points $\mathbf{y}: \mathbf{y}=\mathrm{T}^{*} \mathbf{x}$.

This was achieved by minimizing the mean squared distances between the feature points and the current model shape $\mathrm{x}$, by means of Procrustes analysis [30]. The displacement for each image/model intersection $\overrightarrow{v_{l, j}}$ along the image plane was computed as:

$$
\overrightarrow{\boldsymbol{v}}_{i, j}=\boldsymbol{s}_{i, j}-I_{i, j}^{k}
$$

Where $i$ and $j$ refer to the nodes of the mesh at the position $n_{i}$ and $n_{j}$ whose joining segment intersected the $k^{\text {th }}$ image plane at the point $I_{i, j}^{k}$ and $s_{i, j}$ is the corresponding feature point, computed as defined in the previous section. This displacement was then partitioned between the $n_{i}$ and $n_{j}$ node proportionally to the distance from their image intersection. Finally, the candidate displacement vector $v$ of each $\mathrm{i}^{\text {th }}$ node in the CMR reference system was defined as the average displacement considering all the intersections in which it is involved.

The collection of the displacements for all nodes defined the model update: $\boldsymbol{x}_{n}=\boldsymbol{x}_{n-1}+d \boldsymbol{x}_{n}$, where $\mathrm{dx}_{\mathrm{n}}=$ 
$\left(v_{1}, v_{2}, \ldots v_{\mathrm{n}}\right)$ includes all node displacements computed as in Eq. 6. Consequently, the new shape parameters $\mathbf{b}$ at the $\mathrm{n}^{\text {th }}$ iteration were updated as follows:

$$
\begin{gathered}
\boldsymbol{b}_{n}=\boldsymbol{b}_{n-1}+d \boldsymbol{b}_{n}, \\
d b_{n}=P^{T} * T_{n}^{-1} * d \boldsymbol{x}_{n},
\end{gathered}
$$

where $\mathrm{P}$ represents the matrix describing the transformation between the current model shape $\mathrm{x}$ and the projection of the new shape $\mathrm{x}_{\mathrm{n}+1}$ onto the model coordinate system. As in the standard ASM procedure, the shape parameters $b$ were limited by the statistical characteristics of the shape model:

$$
|\boldsymbol{b}+d \boldsymbol{b}| \leq 3 \sigma
$$

The described steps were applied iteratively, until the mean displacement of all surface nodes was below the CMR inplane spatial resolution $(0.7 \mathrm{~mm})$, considered as threshold value under which the computed model update will not produce a significant change in the mesh.

\subsection{Epicardial segmentation}

The initial pose and scaling of the SSM for epicardial segmentation was automatically given by the final result of the 3D endocardial segmentation in the CMR reference system, from which the same LV model was iteratively deformed to fit the epicardium.

A similar scheme to that described in the previous section for the LV endocardial segmentation was applied according to the ASM fitting algorithm, with the following modification to accommodate/adjust the 3D epicardial shape. The feature point search was performed after sampling all CMR images in the direction connecting the model/image intersections and their centroid, with length fixed empirically to $42 \mathrm{~mm}$ : $14 \mathrm{~mm}$ for the inwards direction (i.e. from the intersections to their centroid) and $28 \mathrm{~mm}$ for the outwards direction (i.e. from the intersections towards the myocardium, Figure 5 a)).

Pixel intensity profiles along the radial samplings were extracted and systematically aligned to compose 2D images constituting the research space (Figure $5 \mathrm{~b}$ )), that were simultaneously processed by means of K-means clustering (Figure $5 \mathrm{c})$ ). As now the interest was in the myocardium/external structures interface, the number of k-means clusters was set equal to 6: one cluster matching the darker signal corresponding to the air in the lungs, three clusters matching 
the medium intensity values corresponding to the myocardial signal and the last two clusters matching the higher intensity values of the blood signal and other external bright structures such as the epicardial fat.

Moreover, a gray-value level was defined as a threshold to reinforce the distinction between clusters describing dark and medium intensity values. This resulted particularly useful when myocardial intensities spanned a wider gray-level range and part of the myocardium would be included in the darker cluster corresponding to the air intensity range, as also observed in [10]. This gray level was empirically set to 0.1 (normalized value), above which pixels should be classified as belonging to the myocardial clusters and not to the air cluster, and vice versa.

Candidate feature points were selected as the interface points between the myocardial clusters and other brighter/darker structures moving from inside the model to outside the model (Figure $5 \mathrm{~d}$ )). A convex-hull filtering was finally applied to this interface boundary, similarly to the approach used for the removal of papillary muscle in the endocardium segmentation step, to remove from the interface boundary cusps or regions with high derivative, that may occur for example at the right ventricle insertion or in close correspondence of the myocardium and external structures with similar intensities (e.g. liver). Feature points were selected as the final myocardium-external structure interface and transformed back in image coordinates to guide the next steps of the model fitting. The AP and MV points manually selected at the initialization step were considered as feature points together with the computed candidates points, to constraint the enlargement of the model in the LV long-axis direction during the affine repositioning through Procrustes analysis. When segmenting the ES frame, the AP was automatically repositioned along the long-axis in the MV to AP direction of an amount equal to the slice-thickness, to roughly compensate for the myocardial thickness at the apical level. At this stage, user interaction was possible to adjust the MV and AP position if necessary. Computation of pose parameters, model parameters and consequent model deformation were iterated according to the ASM algorithm, as described for the endocardium segmentation.

\section{Imaging data and evaluation protocol}

To test the performance of the segmentation, we retrospectively selected 30 patients ( 15 with normal LV function, 10 with coronary artery disease and 5 with dilated cardiomyopathies) that underwent CMR examination at the Centro Cardiologico Monzino. All patients were in sinus rhythm during image acquisition and each of them gave his/her informed consent to the research protocol, approved by the local ethical committee. ECG-gated, steady-state free precession (SSFP) cine-images (GE, $1.5 \mathrm{~T}$, matrix dimension $512 \times 512$ and spacing $0.74 \times 0.74 \mathrm{~mm}$, slice thickness 8 $\mathrm{mm}$, no overlap, no gap) were acquired in the SA view scanning the $\mathrm{LV}$ from atrium to apex and in $2 \mathrm{CH}, 3 \mathrm{CH}$ and $4 \mathrm{CH}$ LA views, as part of the routine CMR examination. ED and ES frames were selected for the analysis. Patients' characteristics are summarized in Table 2. 
An experienced observer manually traced the endocardial (including blood pool and papillary muscles) and epicardial borders from SA and LA CMR images, to be used as reference for comparison. Quantitative analyses were performed as follows.

1) Point-to-surface distance was computed as the $3 \mathrm{D}$ distance from the points constituting the endocardial and epicardial manual reference and the 3D surface resulting from the semi-automatic segmentation.

2) Dice similarity coefficient (Dice $=2|X \cap Y| /|X|+|Y|$, where $X$ and $Y$ are the areas under the contours to compare) and Hausdorff distance (given two definite set of points corresponding to two contours to compare $A=\left\{a_{1}, a_{2}, \ldots, a_{n}\right\}$ and $B=\left\{b_{1}, b_{2}, \ldots, b_{n}\right\}$, Hausdorff $(A, B)=\max (h(A, B), h(B, A)$, where $h(A, B)=$ $\left.\max _{a \in A} \min _{b \in B}\|a-b\|\right)$ between the manual reference contours and the semi-automated contours obtained as the intersection between the 3D triangulated surface and each CMR image plane. These indices were computed separately for endocardium and epicardium and for the ED and ES frame.

3) Clinical measurements derived from contours were computed: i) volumes expressed in ml, computed at ED and ES for both endocardium and epicardium as the area inside the contours multiplied by the slice thickness (Simpson's rule) and ii) myocardial mass, computed as the difference of epicardial and endocardial volumes computed at end-diastole, multiplied by the myocardial density (1.05 $\mathrm{g} / \mathrm{ml}$ [31]). Wilcoxon rank sum test was applied to test the significance of the differences between the methods. Linear regression and Bland-Altman analyses were also applied to evaluate the agreement of the methods.

4) Repeatibility: the same observer was asked to repeat the manual tracings of endocardial and epicardial borders employed as gold standard on 15 patients that were chosen randomly. Variability analysis was then performed by computing intra-class correlation coefficient (ICC) and coefficient of variation $(\mathrm{CoV}=100 * \mathrm{SD} /$ mean $)$ on the point-to-surface errors computed using the original manual tracings and the repeated ones.

\section{Results}

The 3D LV model was built from 435 3DE datasets, including overall 9038 3D LV surfaces, with median 3D volume of $87.5 \mathrm{ml}$ (interquartile range: $58.5 \mathrm{ml}-131 \mathrm{ml}$ ). The segmentation with the proposed ASM approach was then feasible for all CMR datasets, with a stable solution reached for both the endocardial and the epicardial segmentation. An example of the myocardial segmentation in a patient with normal cardiac function is presented in Figure 6: both 3D segmentation (right) and 2D intersections between the 3D models and the CMR image planes that were used to guide the ASM process (left) showed correct detection of the endocardium and the epicardium with smooth shape and realistic 
segmentation in both SA and LA direction. The point-to-surface errors, measuring the distances between the manual reference technique in the CMR images and the 3D segmentation, is shown in the right-bottom panel and confirms the reliability of the 3D segmentation, with some slightly higher errors in this case for the endocardium in the mid apical position.

Quantitative analysis resulted in small point-to-surface distances, with median values ranging from 1.64 to $1.97 \mathrm{~mm}$, Hausdorff distance of about $5 \mathrm{~mm}$ and very Dice similarity coefficient $(>0.84)$, showing a high and local agreement of $3 \mathrm{D}$ segmentation and derived contours compared to the manual reference technique. Results of this quantitative analysis are presented in Table 3 .

On derived clinical parameters, i.e. endocardial and epicardial volumes and LV masses, no significance difference between manual reference and proposed ASM was found $(\mathrm{p}<0.01$, Wilcoxon rank sum test). Linear correlation and Bland-Altmann analysis results are showed in Figures 7-8-9, respectively.

Overall, high and significant correlation coefficient (Spearman rho, $\rho>0.89$ ) were found, with the highest value for ES epicardial volume $(\rho=0.97)$, and slopes very close to 1 (ranging from 0.99 to 1.15 ). Bland-Altman analysis showed a slight systematic overestimation of computed volumes compared to the manual reference (bias $=8.06^{*} \mathrm{ml}$ and $18.81^{*}$ $\mathrm{ml}$ for the endocardium and bias $=5.73 \mathrm{ml}$ and $12.74 * \mathrm{ml}$ for the epicardium; $*$ : $<<0.01$ Wilcoxon test) while a slight underestimation of myocardial mass (bias $=-6.73 \mathrm{~g}, *: \mathrm{p}<0.01$ Wilcoxon test). Limits of agreement were narrower for the endocardium volumes (6.45 to $31.17 \mathrm{ml}$ and -4.09 to $20.21 \mathrm{ml}$ for EDV and ESV, respectively) compared to those of the epicardium (-18.52 to $44.00 \mathrm{ml}$ and -24.49 to $35.94 \mathrm{ml}$ for EDV and ESV, respectively).

An apparent positive correlation can be noticed for ES endocardial and epicardial volumes (Figure 8 and 9, bottom right plot), suggesting that errors increase with increasing volumes. This was then quantified by computing linear regression analysis on the ESV endocardium and epicardial errors, which resulted in moderate correlation (Spearman's rho equal to 0.52 and 0.74 for ESV endocardial error and epicardial error, respectively), but slopes close to zero ( 0.09 and 0.15 for ESV endocardial error and epicardial error, respectively).

Reliability of the analysis is supported by the intra-observer variability analysis of point-to-surface error, which resulted in all cases in high values of ICC (around 0.8$)$ and small values of CoV $(\leq 10.5 \%)$. Detailed results are reported in Table 4.

\section{Discussion}

We have presented in this paper the construction of a statistical model of the LV built from real time 3D echocardiography and its application to segment the LV myocardium in CMR images. 
Our study confirmed that 3DE represents a convenient choice for the training database, being noninvasive, widely used in clinics and allowing the 3D spatio-temporal representation of the cardiac chambers, as also previously suggested [27, 32]. This strategy potentially overcomes the limitation of creating 3D models from interpolated 2D contours from CMR images, which in standard acquisitions are characterized by submillimetric in-plane resolution but slice thickness up to $8 \mathrm{~mm}$. As a consequence, the derived models could resent from interpolated geometry in the longitudinal direction, leading to uncertainties and approximations in particular for the definition of the LV apex and the valvular planes. In [21], this was dealt by combining images with different orientation to build a shape model of the LV. In [17], shape based interpolation was applied to labeled images, to obtain a nearly isotropic voxel resolution. In [15], this limitation was overcome by collecting high resolution CMR datasets, not routinely acquired in clinics and characterized by a lower temporal resolution (20 frames per cardiac cycle), compared to both 3DE and standard CMR cine acquisitions. In our approach, the deformation of the 3DE-based SSM was guided by surface-images intersections as in [11], leading to robust and reliable results for LV segmentation. Moreover, our strategy benefits from the fact that the training set was obtained using commercial software that allows for 4D semi-automatic segmentation. Since it is widely used in clinics and in our institutions, we were able to significantly enlarge the number of the training datasets compared to our preliminary study [27] and furthermore to provide an extensive validation on a CMR population made of normal and pathological subjects.

The choice of considering multiple frames to construct the LV PDM is supported by a previous research [32], where we evaluated the effect of different registration strategies to build the LV PDM and found optimal performances when the PDM included more than one frame within the cardiac cycle.

Quantitative comparison to other studies is not straightforward due to the many differences across the model-based segmentation approaches: imaging technique and number of training images, number and type of patients tested, image quality and resolution, implementation of the ASM and metrics employed for quantitative validation. As discussed by [20], the general trend among model-based segmentation of CMR images is that endocardial errors tend to be the smallest, followed by the epicardial ones $[14,20,33]$. This was not confirmed in our study, in which we found very similar and comparable results between the endocardium and epicardium segmentation, both for contour similarity (point-to-surface distance, Hausdorff and Dice) and for derived clinical parameters (equivalence testing and linear regression analysis), suggesting local and global robustness of the approach.

The Bland-Altman resulted in lower mean errors for the epicardium compared to those of the endocardium, even if with a larger variability. For the ESV endocardium and epicardium error, a slight positive trend was found, suggesting that the proposed approach underestimates the ESV at lower values and overestimate it at the higher values. This might be 
due to the fact that on the ES frame the definition of myocardial borders is more prone to uncertainties, because of the presence of papillary muscles that can appear attached to the endocardium.

As regards the endocardial segmentation, the beneficial effect of increasing the number of patients included in the training set up to 435 subjects should be noticed: compared to our previous study [27] including only 205 subjects, we found narrower limits of agreement for both EDV (6.45 to $31.17 \mathrm{ml} \mathrm{vs} \mathrm{-18} \mathrm{to} 21 \mathrm{ml}$ ) and ESV (4.09 to $20.21 \mathrm{ml} \mathrm{vs}$ 23.5 to $25.5 \mathrm{ml})$

Compared to [10], applying a 3D fuzzy inference matching algorithm to CMR data with the model built from 2D CMR stacks from 53 patients and tested on the ED frame of additional 15 patients, our study showed similar limits of agreement for the EDV (around -28 to $-4 \mathrm{ml}$ ), but slightly broader for the epicardial volumes (around -2 to $27 \mathrm{ml}$ ).On the LV mass measurement, which expresses the combined effect of semi-automatic segmentation of both myocardial borders and is computed on the ED frame, promising results were found, suggesting a consistent joint segmentation of endocardium and epicardium. As regards contour quantification, our approach resulted in point-to-surface distances $(\sim 1.95 \mathrm{~mm}$ and $\sim 1.7 \mathrm{~mm}$ for endocardium and epicardium, respectively) smaller than the SSM-based methods proposed first by [21] ( $\sim 2.3 \mathrm{~mm}$ and $\sim 3 \mathrm{~mm}$ for endocardium and epicardium, respectively) and [9] ( $\sim 2.75 \mathrm{~mm}$ and $\sim 2.63 \mathrm{~mm}$ for endocardium and epicardium, respectively), and more recently by [16] ( 2.6 mm for the endocardium). Furthermore, we achieved a slightly better accuracy compared to the SPASM algorithm [11] $(\sim 1.97 \mathrm{~mm}$ and $\sim 2.32 \mathrm{~mm}$ for endocardium and epicardium, respectively), where also LA and SA images are jointly considered for segmentation. Our approach is independent from a dedicated appearance model. In its original formulation, the ASM is made of both a shape and an appearance model [1], where this latter is responsible of learning grey-level patterns from the training set image data, to be compared against those in the images to be segmented in the fitting step. However, due to the differences in image intensities and contrast of echocardiography and CMR, this would have required an additional collection of manual ground truth contours on CMR images necessary to train the intensity model. For this reason, we have chosen to derive appearance information from of the same CMR processed images exploiting local intensity differences. This is similar to the approach proposed by [10], in which however fuzzy c-means clustering instead of kmeans is adopted.

Our study is supported by a variability analysis, which showed high repeatability of the proposed technique, with slightly better results for ED measures compared to the ES ones, probably due to the easier definition of myocardial borders during the dilation phase in the absence of papillary muscles.

One main novelty of this work is the usability of the same LV PDM created from 3D endocardial surfaces to segment both the endocardium and the epicardium in CMR images. This was driven by the assumption that the shape of the myocardial borders could be statistically described by the same SSM. To the best of our knowledge no previous study 
proposed a similar strategy to segment both myocardial borders, nor the relation between the shapes of endocardium and epicardium has been previously investigated. The adaptation of the LV endocardial model for the epicardium segmentation was successfully achieved by applying geometrical constraints on the apex and base, necessary to limit the expansion of the model in the direction of the LV long axis. This may be intuitively explained by considering the fact that the epicardium and the endocardium are the outer and the inner surfaces of the myocardium. The contraction and dilation throughout the cardiac cycle result mainly in myocardial fiber shortening and thickening in the radial direction, together with torsion and base to apex motion, thus maintaining a comparable shape between endo- and epicardium of the LV. Of note, this approach was also driven by an intrinsic limitation of our statistical model, as the epicardium is not often well visualized by $3 \mathrm{DE}$ and therefore it was not possible to build a separate SSM for each myocardial surface.

One limitation of our study is that in its present formulation, user interaction is needed to select anatomical landmarks in the LA images. This is however a simple procedure that allows the correct first positioning of the LV shape onto the CMR stack. In future research, strategies for automated localization of anatomical landmarks will be investigated. Besides dedicated image processing for landmarks localization, image registration techniques could be adopted, so that the landmarks positions detected in one subject could be propagated to new subjects. However, due to the large variability of the cardiac anatomy, non-rigid registration approaches should be considered in this case.

Another limitation of our study is that the method was applied on a relatively small CMR population (30 patients), half of which showed abnormal LV function. To confirm the clinical value of the approach, the method will be tested in a wider population including normal and failing hearts.

\section{Conclusion}

In conclusions, we have proposed and evaluated a semi-automated and unsupervised method for the segmentation of the LV myocardium in short-axis CMR images using an ASM approach based on a multi-frame model. The training set was constituted of LV surfaces derived from 3DE image analysis, obtained from a heterogeneous clinical population including more than 400 patients. Even though the LV model was built from endocardial surfaces only, it was possible apply it, with some geometrical constraints, to segment both the endocardium and the epicardium in short-axis CMR stacks, in a multi-modality application..

The presented results showed its ability in obtaining a 3D segmentation of the LV borders from CMR images in different cardiac phases (ED and ES). We envisage its use to semi-automatically segment the myocardium in CMR, thus obtaining a realistic 3D representation of the $\mathrm{LV}$ also at the basal and apical level, which can be difficult in clinical CMR due to the anisotropic spacing along the LV direction. Besides a potential more accurate estimate of clinical 
parameters, the obtained endo- and epi-cardial surfaces could be used as basis to build patient-specific models for electro-mechanical simulations.

\section{Conflicts of interest}

The authors declare that they have no conflict of interest.

\section{Ethical approval}

In our institution, each patient is asked before data acquisition to provide informed consent for research activity.

Furthermore, local scientific and ethical committees approved the study. 


\section{References}

1. Heimann T, Meinzer HP (2009) Statistical shape models for 3D medical image segmentation: a review. Med Image Anal 13 (4):543-563.

2. Frangi AF, Niessen WJ, Viergever MA (2001) Three-dimensional modeling for functional analysis of cardiac images: a review. IEEE Trans Med Imaging 20 (1):2-25.

3. Petitjean C, Dacher JN (2011) A review of segmentation methods in short axis cardiac MR images. Med Image Anal 15 (2):169-184

4. Piazzese C, Carminati MC, Pepi M, Caiani EG, (2017). Statistical Shape Models of the Heart: Applications to Cardiac Imaging. In: Zheng G (ed) Statistical Shape and Deformation Analysis. Methods, Implementation and Applications. Academic Press, pp 445-480.

5. Cootes TF, Taylor CJ, Cooper DH, Graham J (1995) Active Shape Models-Their Training and Application. Comput Vis Image Underst 61 (1):38-59.

6. Cootes TF, Edwards GJ, Taylor CJ (2001) Active appearance models. IEEE Transactions on Pattern Analysis and IEEE Trans Pattern Anal Mach Intell (6):681-685.

7. Kaus MR, von Berg J, Weese J, Niessen W, Pekar V (2004) Automated segmentation of the left ventricle in cardiac MRI. Med Image Anal 8 (3):245-254.

8. Medrano-Gracia P, Cowan BR, Ambale-Venkatesh B, Bluemke DA, Eng J, Finn JP, Fonseca CG, Lima JA, Suinesiaputra A, Young AA (2014) Left ventricular shape variation in asymptomatic populations: the MultiEthnic Study of Atherosclerosis. J Cardiovasc Magn Reson 16:56.

9. Mitchell SC, Bosch JG, Lelieveldt BP, van der Geest RJ, Reiber JH, Sonka M (2002) 3-D active appearance models: segmentation of cardiac MR and ultrasound images. IEEE Trans Med Imaging 21 (9):1167-1178.

10. van Assen HC, Danilouchkine MG, Dirksen MS, Reiber JH, Lelieveldt BP (2008) A 3-D active shape model driven by fuzzy inference: application to cardiac CT and MR. IEEE Trans Inf Technol Biomed 12 (5):595-605.

11. van Assen HC, Danilouchkine MG, Frangi AF, Ordas S, Westenberg JJ, Reiber JH, Lelieveldt BP (2006) SPASM: a 3D-ASM for segmentation of sparse and arbitrarily oriented cardiac MRI data. Med Image Anal 10 (2):286-303.

12. Medrano-Gracia P, Cowan BR, Bluemke DA, Finn JP, Kadish AH, Lee DC, Lima JA, Suinesiaputra A, Young AA (2013) Atlas-based analysis of cardiac shape and function: correction of regional shape bias due to imaging protocol for population studies. J Cardiovasc Magn Reson 15:80.

13. Fonseca CG, Backhaus M, Bluemke DA, Britten RD, Chung JD, Cowan BR, Dinov ID, Finn JP, Hunter PJ, Kadish AH, Lee DC, Lima JA, Medrano-Gracia P, Shivkumar K, Suinesiaputra A, Tao W, Young AA (2011) The 
Cardiac Atlas Project--an imaging database for computational modeling and statistical atlases of the heart. Bioinformatics 27 (16):2288-2295.

14. Zhang H, Wahle A, Johnson RK, Scholz TD, Sonka M (2010) 4-D cardiac MR image analysis: left and right ventricular morphology and function. IEEE Trans Med Imaging 29 (2):350-364.

15. Bai W, Shi W, de Marvao A, Dawes TJ, O'Regan DP, Cook SA, Rueckert D (2015) A bi-ventricular cardiac atlas built from 1000+ high resolution MR images of healthy subjects and an analysis of shape and motion. Med Image Anal $26(1): 133-145$.

16. Alba X, Pereanez M, Hoogendoorn C, Swift AJ, Wild JM, Frangi AF, Lekadir K (2016) An Algorithm for the Segmentation of Highly Abnormal Hearts Using a Generic Statistical Shape Model. IEEE Trans Med Imaging 35 (3):845-859.

17. Frangi AF, Rueckert D, Schnabel JA, Niessen WJ (2002) Automatic construction of multiple-object threedimensional statistical shape models: application to cardiac modeling. IEEE Trans Med Imaging 21 (9):11511166.

18. Mitchell SC, Lelieveldt BP, van der Geest RJ, Bosch HG, Reiber JH, Sonka M (2001) Multistage hybrid active appearance model matching: segmentation of left and right ventricles in cardiac MR images. IEEE Trans Med Imaging 20 (5):415-423.

19. Lotjonen JM, Jarvinen VM, Cheong B, Wu E, Kivisto S, Koikkalainen JR, Mattila JJ, Kervinen HM, Muthupillai R, Sheehan FH, Lauerma K (2008) Evaluation of cardiac biventricular segmentation from multiaxis MRI data: a multicenter study. J Magn Reson Imaging 28 (3):626-636.

20. Tobon-Gomez C, Sukno FM, Butakoff C, Huguet M, Frangi AF (2012) Automatic training and reliability estimation for 3D ASM applied to cardiac MRI segmentation. Phys Med Biol 57 (13):4155-4174.

21. Lotjonen J, Kivisto S, Koikkalainen J, Smutek D, Lauerma K (2004) Statistical shape model of atria, ventricles and epicardium from short- and long-axis MR images. Med Image Anal 8 (3):371-386.

22. Hoogendoorn C, Duchateau N, Sanchez-Quintana D, Whitmarsh T, Sukno FM, De Craene M, Lekadir K, Frangi AF (2013) A high-resolution atlas and statistical model of the human heart from multislice CT. IEEE Trans Med Imaging 32 (1):28-44.

23. Ecabert O, Peters J, Walker MJ, Ivanc T, Lorenz C, von Berg J, Lessick J, Vembar M, Weese J (2011) Segmentation of the heart and great vessels in CT images using a model-based adaptation framework. Med Image Anal 15 (6):863-876

24. Ecabert O, Peters J, Schramm H, Lorenz C, von Berg J, Walker MJ, Vembar M, Olszewski ME, Subramanyan K, Lavi G, Weese J (2008) Automatic model-based segmentation of the heart in CT images. IEEE Trans Med 
Imaging 27 (9):1189-1201.

25. Lorenz C, von Berg J (2006) A comprehensive shape model of the heart. Med Image Anal 10 (4):657-670.

26. 1. Peng P, Lekadir K, Gooya A, Shao L, Petersen SE, Frangi AF (2016) A review of heart chamber segmentation for structural and functional analysis using cardiac magnetic resonance imaging. Magn Reson Mater Phy 29 (2):155-195.

27. Caiani EG, Colombo A, Pepi M, Piazzese C, Maffessanti F, Lang RM, Carminati MC (2014) Three-dimensional left ventricular segmentation from magnetic resonance imaging for patient-specific modelling purposes. Europace 16 Suppl 4:iv96-iv101.

28. Neumann A, Lorenz C (1998) Statistical shape model based segmentation of medical images. Comput Med Imaging Graph 22 (2):133-143.

29. Carminati MC, Maffessanti F, Caiani EG (2014) Nearly automated motion artifacts correction between multi breath-hold short-axis and long-axis cine CMR images. Comput Biol Med 46:42-50.

30. Goodall C (1991) Procrustes Methods in the Statistical Analysis of Shape. J R Stat Soc Series B Stat Methodol 53 (2):285-339

31. Schulz-Menger J, Bluemke DA, Bremerich J, Flamm SD, Fogel MA, Friedrich MG, Kim RJ, von KnobelsdorffBrenkenhoff F, Kramer CM, Pennell DJ, Plein S, Nagel E (2013) Standardized image interpretation and post processing in cardiovascular magnetic resonance: Society for Cardiovascular Magnetic Resonance (SCMR) board of trustees task force on standardized post processing. J Cardiovasc Magn Reson 15:35.

32. Piazzese C, Carminati MC, Colombo A, Krause R, Potse M, Auricchio A, Weinert L, Tamborini G, Pepi M, Lang RM, Caiani EG (2016) Segmentation of the left ventricular endocardium from magnetic resonance images by using different statistical shape models. J Electrocardiol 49 (3):383-391.

33. Koikkalainen J, Tolli T, Lauerma K, Antila K, Mattila E, Lilja M, Lotjonen J (2008) Methods of artificial enlargement of the training set for statistical shape models. IEEE Trans Med Imaging 27 (11):1643-1654. 